\title{
Feasibility of non-linear simulation for Field II using an angular spectrum approach
}

\author{
Du, Yigang; Jensen, Jørgen Arendt
}

Published in:

2008 IEEE Ultrasonics Symposium

Link to article, DOI:

10.1109/ULTSYM.2008.0318

Publication date:

2008

Document Version

Publisher's PDF, also known as Version of record

Link back to DTU Orbit

Citation (APA):

Du, Y., \& Jensen, J. A. (2008). Feasibility of non-linear simulation for Field II using an angular spectrum approach. In 2008 IEEE Ultrasonics Symposium (Vol. 1-4, pp. 1314-1317). IEEE. I E E E International Ultrasonics Symposium. Proceedings https://doi.org/10.1109/ULTSYM.2008.0318

\section{General rights}

Copyright and moral rights for the publications made accessible in the public portal are retained by the authors and/or other copyright owners and it is a condition of accessing publications that users recognise and abide by the legal requirements associated with these rights.

- Users may download and print one copy of any publication from the public portal for the purpose of private study or research.

- You may not further distribute the material or use it for any profit-making activity or commercial gain

- You may freely distribute the URL identifying the publication in the public portal 


\title{
Feasibility of non-linear simulation for Field II using an angular spectrum approach
}

\author{
Yigang $\mathrm{Du}^{1,2}$ and Jørgen Arendt Jensen ${ }^{1}$ \\ 1) Center for Fast Ultrasound Imaging, DTU•Elektro, Bldg. 349, \\ Technical University of Denmark, DK-2800 Kgs. Lyngby, Denmark \\ 2) B-K Medical Aps, Mileparken 34, DK-2730 Herlev, Denmark.
}

\begin{abstract}
Simulation of non-linear fields is most often restricted to single element, circularly symmetric sources, which is not used in clinical scanning. To obtain a general and valuable simulation, array transducers of any geometry with any excitation, focusing, and apodization should be modeled. Field II is restricted to simulate these for the linear case and the purpose of this paper is to develop a general frame work for extending it to non-linear simulation. The extension to the non-linear domain is made by using the angular spectrum approach (ASA), where the field is calculated in a plane close to the transducer surface. This calculation is performed using Field II and, thus, includes modeling array transducers of any geometry with any excitation, focusing, and apodization. The propagation in the linear or nonlinear medium is then performed using the angular spectrum approach. The first step in deriving this procedure is to find the accuracy of the approach for linear propagation, where the result can be validated using Field II simulations. The ASA calculations are carried out by 3D fast Fourier transform using Matlab, where $\lambda / 2$ is chosen as the spatial sampling rate to reduce aliasing errors. Zero-padding is applied to enlarge the source plane to a $(4 N-1) \times(4 N-1)$ matrix to overcome artifacts in terms of the circular convolution. The source plane covering an area of $9 \times 9 \mathrm{~mm}^{2}$ with $N=61$ samples along both side, is $0.05 \mathrm{~mm}$ away from a $5 \mathrm{MHz}$ planar piston transducer, which is simulated by Field II. To determine the accuracy, different sampling intervals and zero-paddings are compared and the errors are calculated with Field II as a reference. It can be seen that zero-padding with $4 N-1$ and $\lambda / 2$ sampling can both reduce the errors from $25.7 \%$ to $12.9 \%$ for the near-field and from $18.1 \%$ to $5.8 \%$ for the far-field, and improve the price of an increase in computation time. The angular spectrum approach in combination with Field II opens for the possibility of simulating the non-linear acoustic propagation for any kind of array transducers.
\end{abstract}

\section{INTRODUCTION}

Non-linear imaging is extensively used in the clinic due to its improved image quality [1] [2]. It has been observed that the contrast is significantly better. However, there is still a limited understanding of the inner workings of non-linear imaging [3] [4]. It is very difficult to realistically simulate the non-linear propagation and often it is not understood why the non-linear images are better.

To archive an optimal image quality, it is of great importance to obtain a simulation software, by which array transducers of any arbitrary geometry with any excitation, focusing and apodization can be modeled.

Field II [5] [6] is a program for simulating ultrasound transducer fields and any kind of linear imaging can be simulated as well as realistic images of human tissue.

To expand Field II for the same application into the nonlinear domain, an angular spectrum approach is used to solve the non-linear Westervelt wave equation. A source plane is selected as the initial plane for simulating acoustic propagation, and the second harmonic component will be calculated from the fundamental pressure. In this paper, the linear ASA is investigated with reference to Field II and the accuracy is improved using zero-padding and $\lambda / 2$ sampling, which aim at validating the feasibility and optimizing the implementation of the non-linear simulation for Field II.

\section{THEORY}

\section{A. Westervelt equation}

Non-linear propagation can be described by the Westervelt equation [3]. It is composed of a state equation, a continuity equation, and an Euler force equation [7]

$$
\begin{aligned}
\frac{P}{P_{0}} & =\left(\frac{\rho}{\rho_{0}}\right)^{\gamma} \\
\frac{\partial \rho}{\partial t}+\nabla \cdot(\rho \vec{u}) & =0 \\
\rho_{0} \frac{\partial \vec{u}}{\partial t}+\nabla p & =0
\end{aligned}
$$

where $P_{0}$ and $P$ are the equilibrium pressure and instantaneous pressure, $\rho_{0}$ and $\rho$ are equilibrium density and instantaneous density, $\gamma$ is the rate of specific heats, and $\vec{u}$ is the particle velocity.

One solution to (1) can be expressed by a Taylor expansion

$$
P=P_{0}+\left(\frac{\partial P}{\partial \rho}\right)_{\rho_{0}}\left(\rho-\rho_{0}\right)+\frac{1}{2}\left(\frac{\partial^{2} P}{\partial \rho^{2}}\right)_{\rho_{0}}\left(\rho-\rho_{0}\right)^{2}+\cdots
$$

With the help of the relations $s=\left(\rho-\rho_{0}\right) / \rho$ and $p=P-P_{0}$, (4) can be rewritten as

$$
s=\frac{p}{\rho_{0} c^{2}}-\frac{1}{2}(\gamma-1) \frac{p^{2}}{\rho_{0}^{2} c^{4}} .
$$

For plane waves, the characteristic acoustic impedance is defined as

$$
z=p / u=\rho_{0} c,
$$

where $c=\sqrt{P_{0} \gamma / \rho_{0}}$ is the speed of sound [7]. Thus, combining (2), (3), (5) and (6), the lossless Westervelt equation 


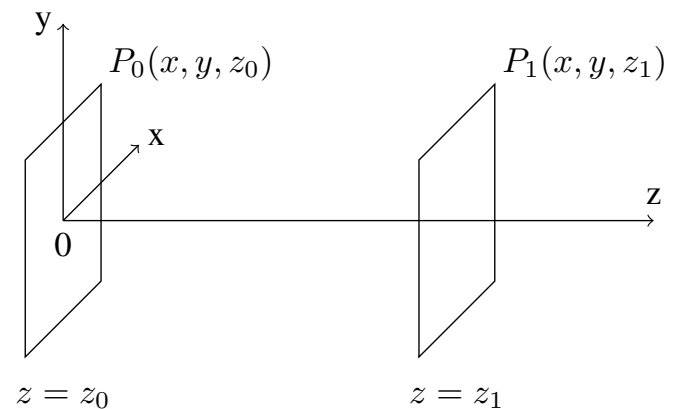

Fig. 1. Schematic view of angular spectrum approach

is expressed as

$$
\left(\nabla^{2}-\frac{1}{c_{0}^{2}} \frac{\partial^{2}}{\partial t^{2}}\right) p=-\frac{\beta}{\rho_{0} c_{0}^{4}} \frac{\partial^{2} p^{2}}{\partial t^{2}}
$$

where $\beta$ is the coefficient of nonlinearity, defined as $\beta=$ $(\gamma+1) / 2$.

\section{B. ASA for linear propagation}

Linear acoustic propagation can be simulated in an efficient way by an angular spectrum approach, by calculating the acoustic pressure in the spatial frequency domain superposing plane waves traveling perpendicular to the original source plane [8]. In Fig. 1, the plane at $z=z_{0}$ is the source plane. The spatial Fourier transform of $P_{0}\left(x, y, z_{0}\right)$ is given by

$$
\hat{P}_{0}\left(k_{x}, k_{y}, z_{0}\right)=\iint P_{0}\left(x, y, z_{0}\right) e^{-j\left(k_{x} x+k_{y} y\right)} d x d y .
$$

The acoustic pressure in the plane at $z=z_{1}$ can be obtained by

$$
P_{1}\left(x, y, z_{1}\right)=\mathbf{F}^{-1}\left\{\hat{P}_{0}\left(k_{x}, k_{y}, z_{0}\right) e^{j k_{z}\left(z_{1}-z_{0}\right)}\right\},
$$

where $\mathbf{F}^{-1}\{\}$ represents the inverse Fourier transform. $k_{x}, k_{y}$ and $k_{z}$ are the wave numbers along the respective axis and satisfy $k=\omega / c=\sqrt{k_{x}^{2}+k_{y}^{2}+k_{z}^{2}}$.

\section{ASA for nonlinear propagation}

The ASA technique was introduced in non-linear acoustics by Alais and Hennion [9]. It was further developed by Landsberger and Hamilton [10], where non-linear generation was investigated from a planar source in solid materials using ASA. In this paper, ASA is applied to solve the Westervelt equation derived in the previous section based on the solution derived by Xiang [11].

With the assumption of $p_{1} \gg p_{2}$, a quasi-linear approximation is applied to solve the lossless Westervelt equation and the sound pressure is expressed as

$$
p=p_{1}+p_{2},
$$

where $p_{1}$ and $p_{2}$ denote the fundamental and second harmonic components of the sound pressure, respectively. Using the separate variable solution [7] [10] [12],

$$
p_{n}(x, y, z, t)=\frac{1}{2} P_{n}(x, y, z) e^{-j n \omega t}+c . c ., \quad n=1,2
$$

where $P_{n}$ is the amplitude of the sound wave, $\omega$ is the fundamental angular frequency given by the source plane and c.c. means the complex conjugate of the preceding term. Substitute (10) and (11) into (7) leads to

$$
\left(\nabla^{2}+k^{2}\right) P_{1}+\left(\nabla^{2}+4 k^{2}\right) P_{2} e^{-j \omega t}=\frac{2 \beta k^{2}}{\rho_{0} c_{0}^{2}} P_{1}^{2} e^{-j \omega t} .
$$

Equation (12) can be divided into two equations since the first term of the left hand side of (12) is not a function of time $t$.

$$
\begin{aligned}
\left(\nabla^{2}+k^{2}\right) P_{1} & =0, \\
\left(\nabla^{2}+4 k^{2}\right) P_{2} & =\frac{2 \beta k^{2}}{\rho_{0} c_{0}^{2}} P_{1}^{2} .
\end{aligned}
$$

The linear equation (13) is solved by (9) and the solution to the non-linear one (14) is derived by taking the $2 \mathrm{D}$ spatial inverse Fourier transform for $P_{1}$ and using the convolution theorem

$$
f * g=\mathbf{F}\left\{\mathbf{F}^{-1}\{f\} \cdot \mathbf{F}^{-1}\{g\}\right\},
$$

where $\mathbf{F}\{\}$ represents the Fourier transform. Then equation (14) can be expressed as

$$
\begin{aligned}
\left(\nabla^{2}+4 k^{2}\right) P_{2}= & \mathbf{F}^{-1}\left\{\frac{\beta k^{2}}{2 \pi^{2} \rho_{0} c_{0}^{2}} \iint \hat{P}_{1}\left(k_{x}^{\prime}, k_{y}^{\prime}, z\right)\right. \\
& \left.\times \hat{P}_{1}\left(k_{x}-k_{x}^{\prime}, k_{y}-k_{y}^{\prime}, z\right) d k_{x}^{\prime} d k_{y}^{\prime}\right\} .
\end{aligned}
$$

An ordinary differential equation with an inhomogeneous integral term is obtained by taking the inverse Fourier transform for $P_{2}$ in (16),

$$
\left(\frac{d^{2}}{d z^{2}}+k_{z 2}^{2}\right) \hat{P}_{2}\left(k_{x}, k_{y}, z\right)=\alpha M\left(k_{x}, k_{y}, z\right),
$$

where

$$
\begin{aligned}
M\left(k_{x}, k_{y}, z\right)= & \iint e^{j\left(z-z_{0}\right)\left(k_{z}^{\prime}+k_{z}^{\prime \prime}\right)} \hat{P}_{1}\left(k_{x}^{\prime}, k_{y}^{\prime}, z_{0}\right) \\
& \times \hat{P}_{1}\left(k_{x}-k_{x}^{\prime}, k_{x}-k_{y}^{\prime}, z_{0}\right) d k_{x}^{\prime} d k_{y}^{\prime}, \\
k_{z 2}= & \sqrt{4 k^{2}-k_{x}^{2}-k_{y}^{2}} \\
\alpha= & \frac{\beta k^{2}}{2 \pi^{2} \rho_{0} c_{0}^{2}} \\
k_{z}^{\prime}= & \sqrt{k^{2}-\left(k_{x}^{\prime}\right)^{2}-\left(k_{y}^{\prime}\right)^{2}} \\
k_{z}^{\prime \prime}= & \sqrt{k^{2}-\left(k_{x}-k_{x}^{\prime}\right)^{2}-\left(k_{y}-k_{y}^{\prime}\right)^{2}} .
\end{aligned}
$$

Finally, the solution to (17) gives [10] [11]

$$
\begin{aligned}
\hat{P}_{2}\left(k_{x}, k_{y}, z\right)= & \frac{\beta k^{2}}{2 \pi^{2} \rho_{0} c_{0}^{2}} \iint \frac{\hat{P}_{1}\left(k_{x}^{\prime}, k_{y}^{\prime}, 0\right)}{k_{z 2}-\left(k_{z}^{\prime}+k_{z}^{\prime \prime}\right)^{2}} \\
& \times \hat{P}_{1}\left(k_{x}-k_{x}^{\prime}, k_{y}-k_{y}^{\prime}, 0\right) \\
& \times\left(e^{j z\left(k_{z}^{\prime}+k_{z}^{\prime \prime}\right)}-e^{j k_{z 2} z}\right) d k_{x}^{\prime} d k_{y}^{\prime} .
\end{aligned}
$$

This is the exact final ASA equation for simulating the nonlinear acoustic field. 


\section{METHOD}

So far, a non-linear propagation model is set up by the Westervelt equation and that is solved by the angular spectrum approach. From the solution (23), the second harmonic wave pressure in any position can be obtained as long as the fundamental pressure is known in the source plane, which can be generated by Field II. To make sure the angular spectrum approach can work with Field II, the first step is to simulate the linear acoustic field by using (9) to find the best way to improve the accuracy. The linear simulation of ASA can be compared with Field II in any position with any arbitrary source plane. The fast Fourier transform is applied to this simulation by Matlab and the errors are calculated with reference to Field II.

\section{RESUlts}

The linear acoustic field simulation for a planar piston transducer with the center frequency of $5 \mathrm{MHz}$ and $4 \mathrm{~mm}$ diameter is generated by using Field II. The source plane is $0.05 \mathrm{~mm}$ away from the surface of the transducer due to the singularity at $z=0$. In the beginning, $61 \times 61$ samples with $\lambda / 2$-spacing covering an area of $9 \times 9 \mathrm{~mm}^{2}$ is used as the source plane to avoid the aliasing. Zero-padding is applied to enlarge the source plane twice to overcome artifacts in terms of circular convolution. This is shown in Fig. 2(a), where the calculated plane is $10 \mathrm{~mm}$ away from the source along the z-axis. The values are calculated in the temporal frequency domain by the 3D fast Fourier transform using Matlab. The amplitude at the center line of the plane are shown at a frequency of $5 \mathrm{MHz}$ and the mean error is calculated by

$$
\text { mean error }=\sum_{i=1}^{N}\left|\left(P_{A S A(i)} / P_{\text {Field_II }(i)}-1\right)\right| / N,
$$

where $P_{A S A(i)}$ and $P_{\text {Field_II(i) }}$ are the pressures calculated by the ASA and Field II for the $i$ th point, and $N$ is the number of points. After using zero-padding by 4 , the mean error is reduced from $25.7 \%$ to $12.9 \%$ as shown in Fig. 2(b).

In the far field, the improvement is more obvious when zero-padding is increased as shown in Fig. 3. The mean error is reduced to $1.6 \%$. Furthermore, $\lambda / 4$ is used as the spatial sampling interval to see whether the better accuracy for eliminating the aliasing can be obtained. The complete results are shown in Table. I. Consequently, only for $z=10 \mathrm{~mm}$ and $2 N-1$, the accuracy of $\lambda / 4$ is improved obviously comparing to $\lambda / 2$ as shown in Fig. 2(c).

\begin{tabular}{cccccc} 
& $2 \mathrm{~N}-1$ & $4 \mathrm{~N}-1$ & $6 \mathrm{~N}-1$ & $8 \mathrm{~N}-1$ & $10 \mathrm{~N}-1$ \\
\hline Near-field $(z=10 \mathrm{~mm})$ & & & & \\
$\lambda / 2$ & $25.7 \%$ & $12.9 \%$ & $10.4 \%$ & $9.8 \%$ & $10.1 \%$ \\
$\lambda / 4$ & $14.6 \%$ & $12.0 \%$ & $9.6 \%$ & $9.7 \%$ & $9.5 \%$ \\
\hline $\begin{array}{l}\text { Far-field }(z=100 \mathrm{~mm}) \\
\lambda / 2\end{array}{ }^{18.1 \%}$ & $5.8 \%$ & $4.2 \%$ & $4.1 \%$ & $1.6 \%$ \\
$\lambda / 4$ & $17.5 \%$ & $6.2 \%$ & $2.9 \%$ & $3.2 \%$ & $3.8 \%$ \\
\hline
\end{tabular}

TABLE I

MEAN ERRORS WITH DIFFERENT ZERO-PADDINGS AND SPATIAL SAMPLING RATES BETWEEN THE SIMULATIONS OF FIELD II AND ASA

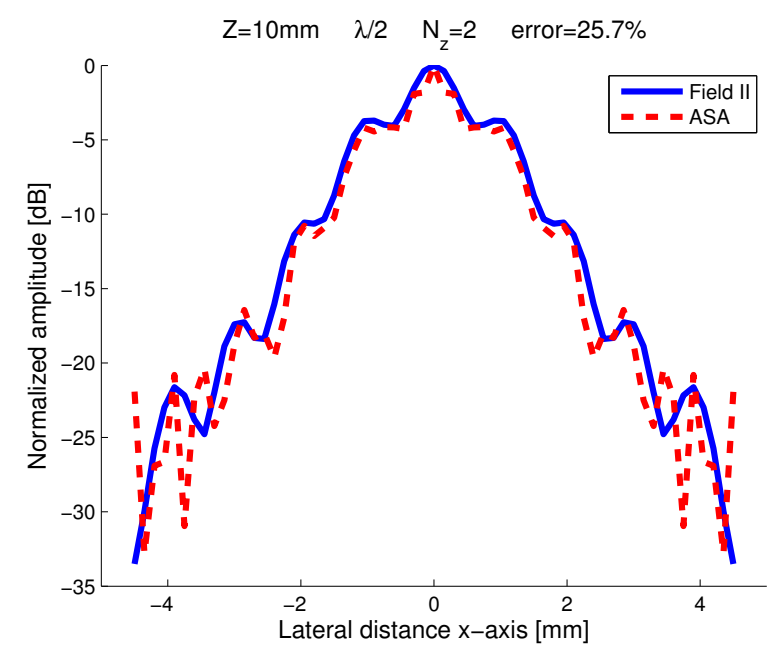

(a) Zero-padding $=2$

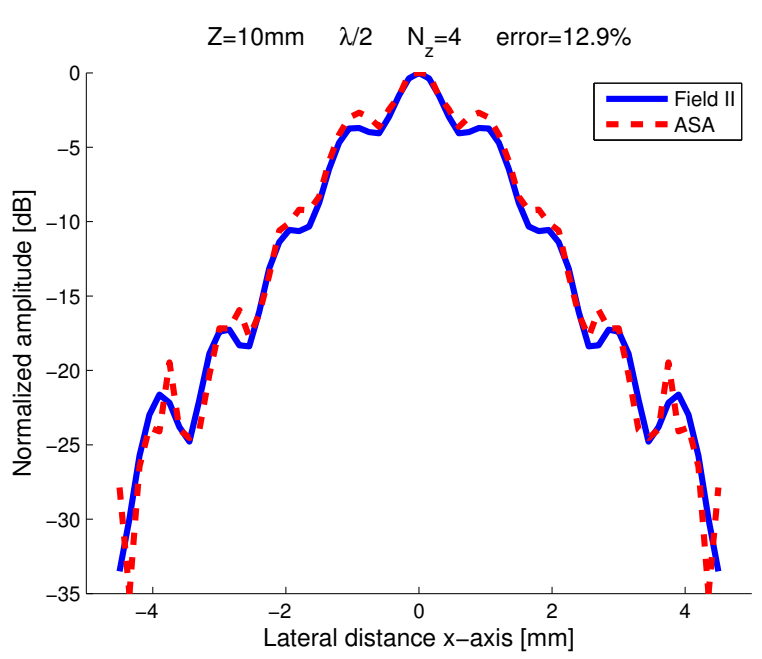

(b) Zero-padding $=4$

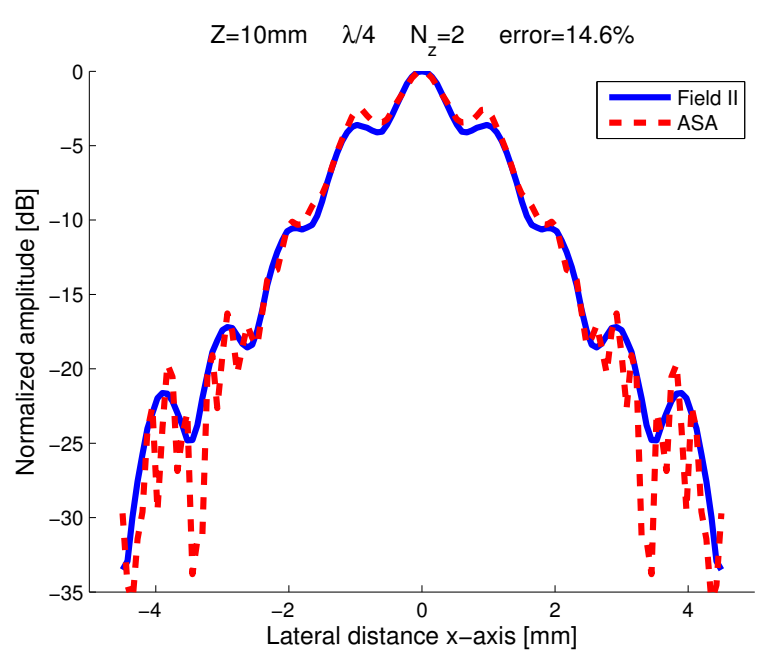

(c) Zero-padding $=2, \lambda / 4$

Fig. 2. Near-field comparison between the simulations of Field II and ASA using different zero-paddings for (a),(b) and $\lambda / 4$ for (c). 


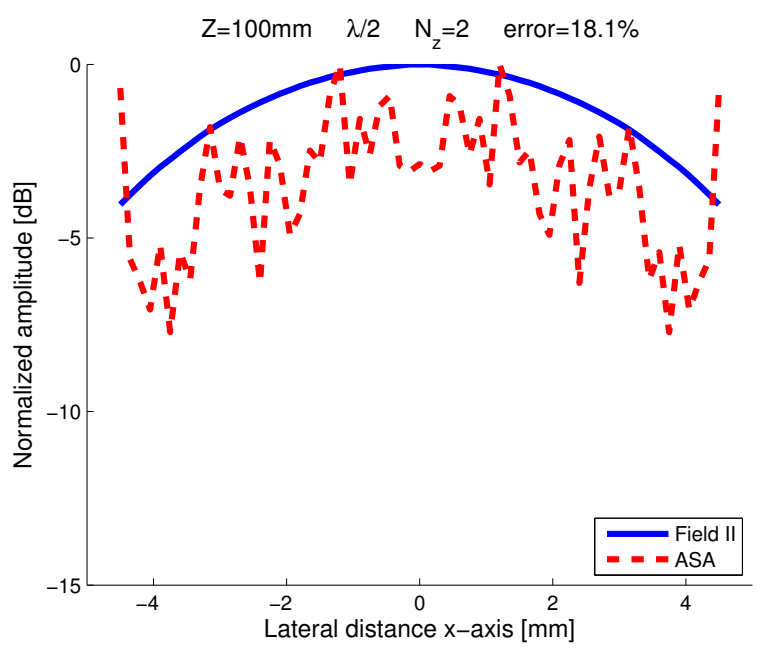

(a) Zero-padding $=2$

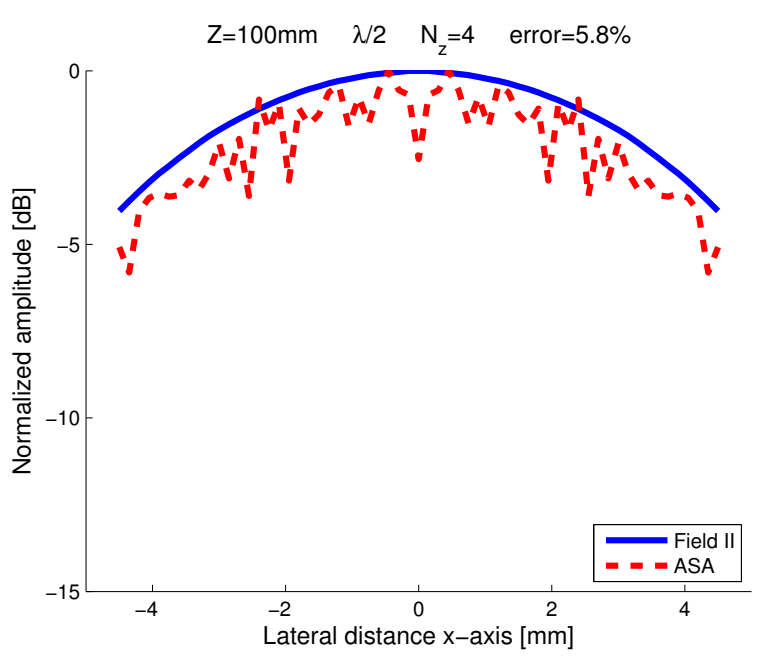

(b) Zero-padding $=4$

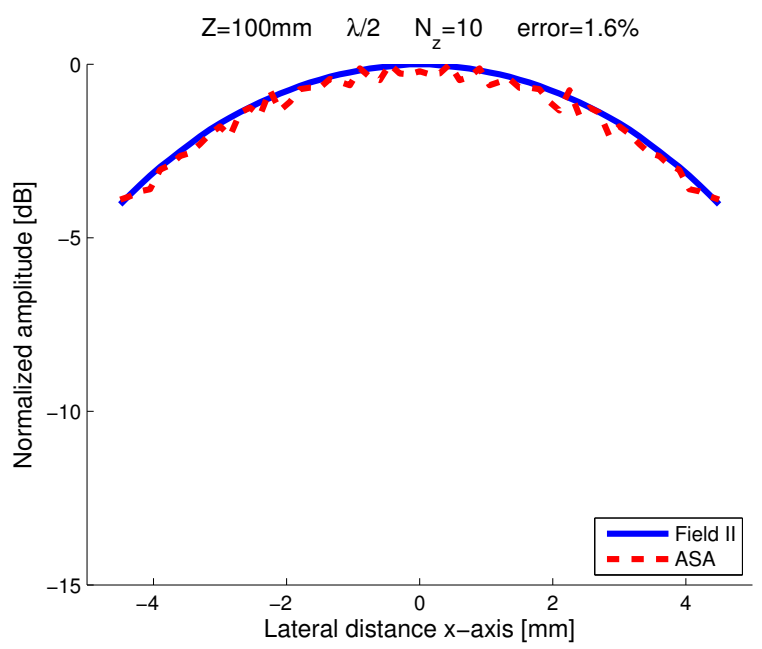

(c) Zero-padding $=10$

Fig. 3. Far-field comparison between the simulations of Field II and ASA using different zero-paddings.

\section{CONCLUSIONS}

The non-linear acoustic field was set up by the Westervelt equation, to which an analytical solution was obtained in this paper using the angular spectrum approach. Using Field II any arbitrary shaped, apodized, and excited ultrasound transducers can be modeled to generate any arbitrary source resulting in possible implementation of a general non-linear acoustic field simulation. The ASA for solving the linear acoustic field is validated by Field II. The agreement of the comparison between the simulations of Field II and ASA is obtained and the accuracy can be improved by using the different zeropaddings and spatial sampling rates. The angular spectrum approach in combination with Field II opens for the possibility of simulating the non-linear acoustic propagation for any kind of array transducers.

\section{FUTURE WORK}

The implementation of (23) for the non-linear propagation will be carried out with the comparison of the experimental measurements of the non-linear acoustic signals in the future work. Thus, the new frame work of the non-linear field will be established by the angular spectrum approach and has the application for simulating any kind of array transducer as long as the linear acoustic source is simulated by the current version of Field II.

\section{ACKNOWLEDGMENT}

This work was supported by grant 9700883,9700563 and 2604-0024 from the Danish Science Foundation and by B-K Medical Aps, Denmark.

\section{REFERENCES}

[1] D. H. Simpson, T. C. Chien, and P. N. Burns, "Pulse inversion doppler: a new method for detecting nonlinear echoes from microbubble contrast agents," IEEE Trans. Ultrason., Ferroelec., Freq. Contr.,, vol. 46, no. 2 pp. 372-382, 1999.

[2] M. A. Averkiou, "Tissue harmonic imaging," in 2000 IEEE Ultrason. Symp.,, 2000, vol. 2, pp. 1563-1572.

[3] M. F. Hamilton and D. T. Blackstock, Nonlinear Acoustics: Theory and Application, Academic Press, 1998.

[4] R. T. Beyer, Nonlinear Acoustics, Acoustical Society of America, 1997.

[5] J. A. Jensen and N. B. Svendsen, "Calculation of pressure fields from arbitrarily shaped, apodized, and excited ultrasound transducers," IEEE Trans. Ultrason., Ferroelec., Freq. Contr., vol. 39, pp. 262-267, 1992.

[6] J. A. Jensen, "Field: A program for simulating ultrasound systems," in Medical \& Biological Engineering \& Computing, 1996, vol. 34, pp. 351-353.

[7] A. B. Coppens L. E. Kinsler, A. R. Frey and J. V. Sanders, Fundamentals of Acoustics, John Wiley and Sons, fourth edition, 2000.

[8] J. W. Goodman, Introduction to Fourier Optics, McGraw-Hill, New York, 1996.

[9] P. Alais and P. Y. Hennion, "A fourier theory of the nonlinear interaction of acoustical beams in absorbing fluid. the special case of parametric emission," Acustica, vol. 43, 1979.

[10] B. J. Landsberger and M. F. Hamilton, "Second-hamonic generation in sound beams reflected from, and transmitted through, immersed elastic solids," JASA, vol. 109, no. 2, pp. 488-500, 2001.

[11] Y. Xiang, Statistical Model of Beam Distortion by Tissue Inhomogeneities in Tissue Harmonic Imaging, Ph.D. thesis, The University of Texas at Austin, December 2004

[12] Y. Xiang and M. F. Hamilton, "Angular spectrum decomposition analysis of second harmonic ultrasound propagation and its relation to tissue harmonic imaging," NDE, June 192006 